\title{
Durability of mortars modified by the effect of combining SPA polymers and supplementary cementitious materials
}

\section{Durabilité des mortiers modifiés par l'effet de la combinaison de Polymère et des matériaux cimentaires supplémentaires}

\author{
B. Belbachir ${ }^{1,2}$, A.S. Benosman ${ }^{1,2,3^{*}}$, H. Taïbi ${ }^{1}$, M. Mouli ${ }^{2}$, Y. Senhadji ${ }^{2,4}$, M. Belbachir ${ }^{1}$ \\ ${ }^{1}$ Faculté des Sciences Exactes et Appliquées, Laboratoire de Chimie des Polymères, Université d'Oran 1, \\ Ahmed Benbella, BP 1524, El Mnaouer, Oran 31000; Algérie. \\ ${ }^{2}$ Département de Génie Civil, Laboratoire des Matériaux LABMAT, ENPO Maurice Audin, Oran 31000 , Algérie. \\ ${ }^{3}$ Ecole Supérieure des Sciences Appliquées, ESSAT, Tlemcen, Algérie. \\ ${ }^{4}$ Faculté des Sciences Techniques, Université Mustapha Stambouli, Mascara, Algérie.
}

\begin{abstract}
Nowadays, the major concern of professionals in the field of building materials is to improve the properties induced by the addition of different additives (polymers) and mineral additions (Supplementary Cement Materials SCMs) and to eventually adapt them to a particular application. This race towards performance has resulted in mortar formulations that are increasingly complex and rich in diversified additions. This is an industry-friendly practice since it generally yields a mortar modified by the combination of a polymer and SCMs, at low cost and low environmental impact, with an improved sustainability in the long term. In order to improve the durability of SCM-modified repair mortars, it seemed interesting to evaluate the influence of adding the styrene polyacrylic (SPA) Latex on the properties of these mortars when exposed to aggressive media such as acids. Composite mortars based on pozzolanic mineral additions, containing different levels of $0.5 \%, 1 \%$ and $2 \%{ }^{\mathrm{w}}$ latex, were stored in acid solutions, for various periods of immersion. The analysis of the microstructure of these mortars, after exposure to acid attack, was carried out by FTIR spectroscopy. The results obtained allowed to demonstrate the beneficial effect of adding the SPA polymer and the pozzolanic additions to the modified materials and to show their improved resistance to acid attacks, such as $\mathrm{HNO}_{3}$ and $\mathrm{H}_{3} \mathrm{PO}_{4}$ solutions at $8 \%$. The best durability properties of the mortars modified by the combination of the polymer and the additional cementitious materials observed in this study indicate a longer service life of the repaired structure when using this type of Latex-modified repair materials.
\end{abstract}

Résumé. Le souci majeur des professionnels dans le domaine des matériaux de construction est d'améliorer les propriétés induites par l'ajout des différents adjuvants (polymères) et des additions minérales (Matériaux Cimentaires Supplémentaires SCMs) et parfois de les adapter à une application particulière. Cette course à la performance s'est traduite par des formulations de mortiers de plus en plus complexes et riches de ces différents ajouts. Cette pratique est favorable à l'industrie, donnant généralement un mortier modifié par l'effet de la combinaison de l'ajout d'un polymère et des SCMs à faible coût, et à faible impact environnemental, avec l'amélioration de la durabilité à long terme. En vue d'améliorer la durabilité des mortiers modifiés de réparation à base des SCMs, on a évalué l'influence de l'ajout du Latex polyacrylique styrol (SPA) sur les caractéristiques de ces derniers vis-à-vis des milieux agressifs tels que les acides. Des mortiers composites avec des bases minérales pouzzolaniques et contenant différents taux de latex $0.5 \%, 1 \%$ et $2 \% \mathrm{w}$ sont conservés dans les solutions acides pour diverses périodes d'immersion. L'analyse de la microstructure de ces mortiers après exposition à l'attaque acide est effectuée par spectroscopie IRTF. Les résultats obtenus permettent de mettre en évidence l'effet bénéfique de l'ajout du polymère SPA et des additions pouzzolaniques dans les matériaux modifiés et sur la résistance aux attaques acides telles que $\mathrm{HNO}_{3}$ et $\mathrm{H}_{3} \mathrm{PO}_{4}$. Or, les meilleures propriétés de durabilité des mortiers modifiés par l'effet de la combinaison de polymère et des matériaux cimentaires supplémentaires observés dans cette étude, indiquent une plus longue durée de vie de la structure réparé en utilisant ce type de matériaux de réparation modifiés par des Latex. 


\section{Introduction}

Au cours de ces dernières décennies la formulation des mortiers composites a été profondément modifiée par l'introduction de multiples adjuvants [1]. Et depuis bien longtemps, la volonté d'optimisation des propriétés des matériaux cimentaires a poussé les formulations à incorporer divers adjuvants organiques ou minéraux dans leur composition [2]. Les adjuvants polymères sont depuis plusieurs dizaines d'années largement utilisés en génie civil et connaissent un accroissement de leur emploi, du fait de la diversité des propriétés qu'ils peuvent offrir $[1,3,4]$.

Le souci majeur des professionnels dans le domaine des matériaux composites de construction est d'améliorer les propriétés induites par l'ajout des différents adjuvants et des additions minérales (SCMs) et parfois de les adapter à une application particulière. Cette course à la performance s'est traduite par des formulations de mortiers composites de plus en plus complexes et riches de ces différents ajouts (ultrafines, polymères, SCMs [5], etc...). L'utilisation de résidus industriels récupérés et recyclés, tels que les ajouts cimentaires et les gisements de ressources naturelles tels que la pouzzolane et le calcaire, comme produits de remplacement partiel du ciment Portland dans le béton, permet de réduire les émissions des gaz à effet de serre $\mathrm{CO}_{2}$ et se traduit par la fabrication d'un béton ou mortier non polluant et durable sur le plan environnemental. Cet accroissement du nombre de constituants est, d'un point de vue pratique, très avantageux car il conduit généralement, à l'amélioration très significative des performances mécaniques, physiques et durabilité [6].

Le but de cette étude est de présenter les résultats d'un travail de recherche portant sur la durabilité des mortiers composites à base de l'effet de la combinaison de l'ajout du polymère SPA et des SCMs telles que la pouzzolane naturelle et la fumée de silice, comme substituant au ciment et ceci pour la réalisation des mortiers de réparation conçus au développement durable. Certaines proportions clés sont également étudiées, contrairement à ce qui a été entrepris dans les travaux antérieurs [5,7-12]. Un complément d'information sur la microstructure a été effectué par la spectrométrie IRTF.

\section{Conditions expérimentales}

Les mortiers composites modifiés par ajout de polymère, ont été réalisés à base d'additions minérales ( $10 \%$ de pouzzolane et $5 \%$ de fumée de silice), avec des teneurs en SPA différentes, tel que : $\mathrm{P} / \mathrm{C}=0,0.5$ et $2 \%$. Les rapports pondéraux utilisés expérimentalement sont $($ ciment $/$ sable $)=1 / 3$ et $($ eau $/$ ciment $)=0.5$ constant pour tous les mortiers. On a utilisé un adjuvant superplastifiant (SP) réducteur d'eau type SIKA ${ }^{\circledR}$ Viscocrète ${ }^{\circledR}$ tempo 12 (Tableau 1).

Les échantillons d'essais ont été confectionnés selon la norme NF P15-403 [13] dans des moules prismatiques $\left(4 \times 4 \times 16 \mathrm{~cm}^{3}\right)$ et vibrés à l'aide d'une table à choc (NF EN 196-1 [14]). Les moules contenant les spécimens sont couverts de film de plastique et conservés dans l'environnement du laboratoire sous une $\mathrm{T}^{\circ} \mathrm{C}$ de $20^{\circ} \pm 1^{\circ} \mathrm{C}$ et une humidité relative d'environ $50 \pm 5 \%$.

Tableau 1. Formulations des différents mortiers modifiés.

\begin{tabular}{|c|c|c|c|c|c|c|}
\hline \multirow[t]{2}{*}{ Code } & \multirow{2}{*}{$\begin{array}{l}\text { Ciment } \\
(\%)\end{array}$} & \multicolumn{2}{|c|}{$\begin{array}{l}\text { SCMs } \\
(\%)\end{array}$} & \multirow{2}{*}{$\begin{array}{l}\text { SPA } \\
(\%)\end{array}$} & \multirow[t]{2}{*}{$\mathrm{E} / \mathrm{C}$} & \multirow{2}{*}{$\begin{array}{l}\text { SP } \\
(\%)\end{array}$} \\
\hline & & $\mathbf{P z}$ & FS & & & \\
\hline Témoin & 100 & - & - & - & 0.5 & 1.3 \\
\hline $\mathrm{PZ}_{10}$ & 90 & 10 & - & - & 0.5 & 1.3 \\
\hline $\mathrm{FS}_{5}$ & 95 & - & 5 & - & 0.5 & 1.3 \\
\hline $\mathrm{Pz}_{10} \mathrm{FS}_{5}$ & 85 & 10 & 5 & - & 0.5 & 1.3 \\
\hline $\mathrm{Pz}_{10} \mathrm{FS}_{5} \mathrm{Pl}_{0.5}$ & 84.5 & 10 & 5 & 0.5 & 0.5 & 1.3 \\
\hline $\mathrm{Pz}_{10} \mathrm{FS}_{5} \mathrm{Pl}_{2}$ & 83 & 10 & 5 & 2 & 0.5 & 1.3 \\
\hline
\end{tabular}

Le démoulage est effectué après 24 heures et les échantillons sont conservés dans une eau basique durant 24 heures. Ensuite, les spécimens sont retirés de cette eau et conservés jusqu'aux 60 jours dans une salle de conservation à $\mathrm{T}^{\circ} \mathrm{C}$ et hygrométrie $\left(20^{\circ} \pm 1^{\circ} \mathrm{C}, \mathrm{HR}=50 \pm 5\right.$ $\%$; milieu référence). Les échantillons dont les dimensions prismatiques $40 \times 40 \times 50 \mathrm{~mm}^{3}$, sont pesés pour déterminer Mo, puis ils sont immergés dans deux différentes solutions acides suivantes : solution d'acide nitrique, $\mathrm{HNO}_{3}$ à $8 \%$ et la solution d'acide orthophosphorique, $\mathrm{H}_{3} \mathrm{PO}_{4}$ à $8 \%$. Le choix s'est porté sur ces deux acides car il s'agit des acides majoritaires trouvés dans les zones industrielles.

La résistance des échantillons immergés vis-à-vis des attaques acides, est déterminée selon la norme ASTM C267-01 [15]. Des analyses IRTF ont été effectuées pour les croutes des échantillons immergés et soumis ou non à l'attaque acide.

\subsection{Le ciment}

Il s'agit d'un ciment Mâtine provenant de l'usine CIBA du groupe LAFARGE sise à OGGAZ (Mascara /Algérie). Ce ciment a une finesse de $4220 \mathrm{~cm}^{2} / \mathrm{g}$, de densité absolue 3.09 et d'une résistance à la compression de $48 \mathrm{MPa}$ à 28 jours. Les compositions chimiques du ciment et minéralogiques de son clinker sont données dans les tableaux 2 et 3 .

Tableau 2. Composition chimique élémentaire du ciment, la pouzzolane et la fumée de silice.

\begin{tabular}{|c|c|c|c|c|c|c|c|c|}
\hline Eléments & $\mathbf{S i O}_{2}$ & $\mathbf{A l}_{2} \mathbf{O}_{3}$ & $\mathbf{F e}_{2} \mathbf{O}_{3}$ & $\mathbf{C a O}$ & $\mathbf{M g O}$ & $\mathbf{S O}_{3}$ & $\mathbf{K}_{2} \mathbf{O}$ & LOI \\
\hline $\mathbf{C M}$ & 17.954 & 4.071 & 2.750 & 58.15 & 1.768 & 2.428 & 0.661 & 10.72 \\
\hline $\mathbf{P z}$ & 46.101 & 16.442 & 9.140 & 9.057 & 5.341 & 0.059 & 1.338 & 7.110 \\
\hline $\mathbf{F S}$ & 94.609 & 0.320 & 1.212 & 0.434 & 0.385 & 0.104 & 0.527 & 1.980 \\
\hline
\end{tabular}

Tableau 3. Composition minéralogique du clinker (\%).

\begin{tabular}{|c|c|c|c|c|}
\hline Eléments & $\mathrm{C}_{3} \mathrm{~S}$ & $\mathrm{C}_{2} \mathrm{~S}$ & $\mathrm{C}_{3} \mathrm{~A}$ & $\mathrm{C}_{4} \mathrm{AF}$ \\
\hline Pourcentage (\%) & 64 & 15 & 8 & 12.16 \\
\hline
\end{tabular}

\subsection{La pouzzolane naturelle "Pz"}

La pouzzolane naturelle, qui est une larve rougeâtre extraite du gisement de Bouhamidi région Béni-saf (Nord-ouest de l'Algérie). Elle a une masse volumique absolue mesurée de $2670 \mathrm{Kg} / \mathrm{m}^{3}$. La pouzzolane est 
broyée ensuite tamisée à travers un tamis de $80 \mu \mathrm{m}$, la poudre résultante donne une surface spécifique de 300 $\mathrm{m}^{2} / \mathrm{Kg}$. Les résultats des analyses chimiques sont présentés dans le tableau 1.

\subsection{La fumée de silice "FS"}

La fumée de silice, comme poudre amorphe de silice extrêmement fine, est un sous-produit de la fabrication du silicium ou des silico-alliages. L'addition qu'on a utilisée dans nos essais, est fournie par l'entreprise TECKNACHEM (Sidi Bel-Abbes/ Algérie). Sa composition chimique est donnée dans le tableau 1.

\subsection{Polyacrylique styrol latex "SPA"}

Le polymère latex utilisé pour la modification de nos mortiers, se présente sous forme de dispersion aqueuse de couleur blanche laiteuse, nommé TEKWELD fourni par l'entreprise TECKNACHEM, à haute densité et stable en milieu alcalin.

\subsection{Le sable}

C'est un sable concassé de fraction $0 / 5$ provenant de la carrière de Nedroma (nord-ouest/Algérie). Afin d'avoir une granulométrie conforme au fuseau du sable normalisé, on a procédé à une correction granulaire de ce sable par un sable marin originaire de Tergua (Aintémouchent /Algérie). Le sable corrigé est $60 \%$ sable de carrière, $40 \%$ sable de mer.

\section{Résultats et discussion}

\subsection{Attaque par l'acide nitrique $\left(\mathrm{HNO}_{3}\right)$}

La Figure 1 exhibe les résultats des pertes de masses pour les spécimens exposés à une solution d'acide $\mathrm{HNO}_{3}$ à $8 \%$. Ces échantillons n'ont pas la même tendance de détérioration, particulièrement avec le mortier $\mathrm{Pz}_{10}$ et le composite $\mathrm{Pz}_{10} \mathrm{FS}_{5} \mathrm{Pl}_{2}$. Après 7 jours, la différence devient tout à fait sensible, elle est supérieure à $20 \%$.

Après 77 jours d'immersion, on constate une perte de masse permanente pour l'ensemble des mortiers. En comparant ces composites, on observe que le mortier témoin et le $\mathrm{Pz}_{10}$ présentait une perte de masse très importante, elle est de $35 \%$ et $42.5 \%$, respectivement. Tandis que les composites modifiés $\mathrm{Pz}_{10} \mathrm{FS}_{5} \mathrm{Pl}_{0.5}$ et $\mathrm{Pz}_{10} \mathrm{FS}_{5} \mathrm{Pl}_{2}$, comparés aux autres mortiers, présentent une grande aptitude à résister à l'attaque acide $\mathrm{HNO}_{3}$, avec des pertes de masse de $17.6 \%$ et $15.2 \%$, respectivement.

A partir de ces résultats, on remarque l'effet bénéfique de la combinaison de l'ajout du polyacrylique styrol (SPA) et des SCMs, ce qui minimise la pénétration des agents agressifs. On a par conséquence résistance et durabilité accrues dans le cas de l'acide nitrique.

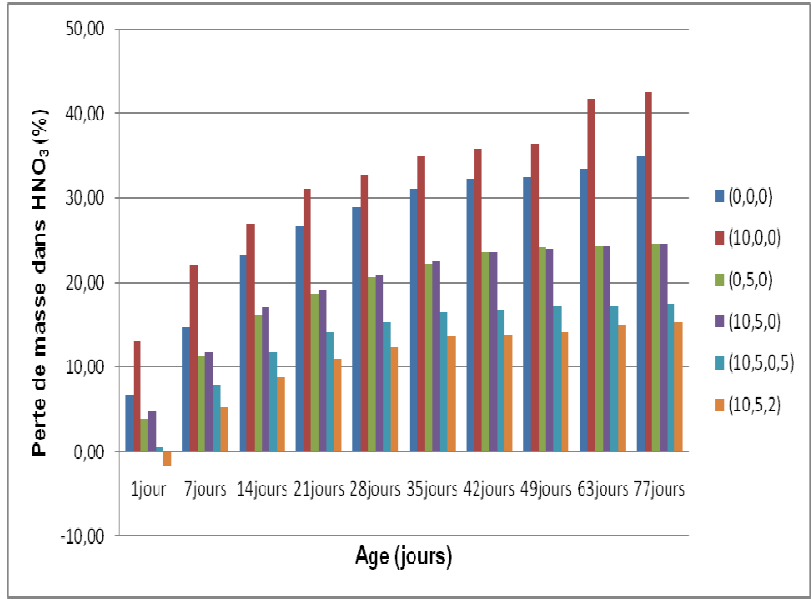

Fig. 1. Effet de la combinaison de l'ajout du polyacrylique styrol et des $\mathrm{SCMs}$ sur l'attaque par $\mathrm{HNO}_{3}$ des mortiers composites.

\subsection{Attaque par l'acide phosphorique $\left(\mathrm{H}_{3} \mathrm{PO}_{4}\right)$}

Les résultats de la perte de masse des spécimens exposés à la solution d'acide phosphorique à $8 \%$, pour une durée de 1 à 49 jours, sont exhibés dans la Figure 2.

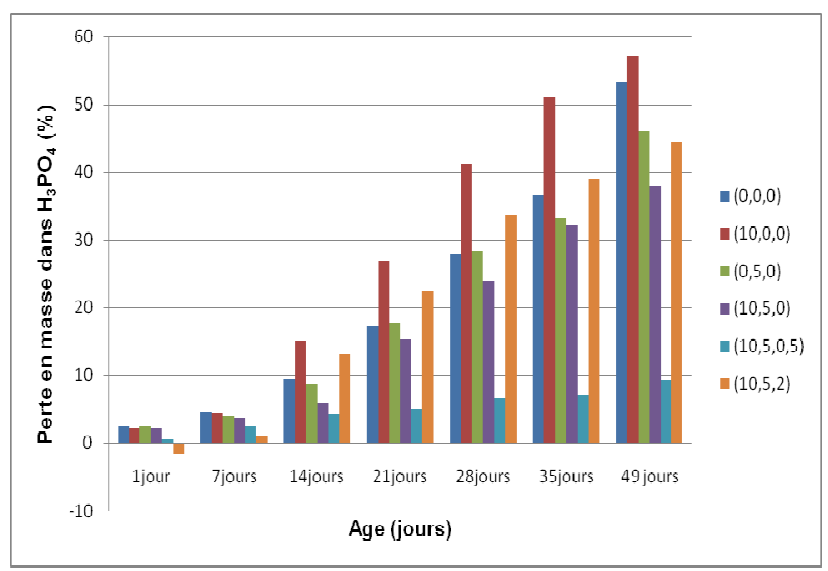

Fig. 2. Effet de la combinaison de l'ajout du SPA et des SCMs sur l'attaque par $\mathrm{H}_{3} \mathrm{PO}_{4}$ des mortiers composites.

A partir de ces résultats, on observe une perte de masse continue légèrement pour tous les mortiers composites à partir de 7 jours.

Après 14 jours d'immersion, on constate une différence de tendance de détérioration, particulièrement avec le mortier $\mathrm{Pz}_{10}$ et le composite $\mathrm{Pz}_{10} \mathrm{FS}_{5} \mathrm{Pl}_{2}$ (supérieure à $10 \%$ ). Tandis que pour le composite $\mathrm{Pz}_{10} \mathrm{FS}_{5} \mathrm{Pl}_{0.5}$ est inférieure à $4 \%$.

Après 28 jours à 49 jours d'immersion, le mortier témoin, le mortier $\mathrm{Pz}_{10}$ et le composite $\mathrm{Pz}_{10} \mathrm{FS}_{5} \mathrm{Pl}_{2}$ présentent la grande perte de masse comparée aux autres. Quoique pour le composite $\mathrm{Pz}_{10} \mathrm{FS}_{5} \mathrm{Pl}_{0.5}$ est inférieure aux autres. On note une perte de masse de $53 \%$ pour le mortier témoin, de $57 \%$ pour $\mathrm{Pz}_{10}$ et de $44 \%$ pour le composite avec ajout de $2 \%$ de SPA. Par contre, on a seulement une perte de masse qui ne dépasse pas les $9 \%$ pour le composite $\mathrm{Pz}_{10} \mathrm{FS}_{5} \mathrm{Pl}_{0.5}$ à 49 jours d'immersion. Dans cette attaque d'ortho-phosphorique, la diminution de la masse est due à la dissolution des hydrates produits 
qui mènent à des mortiers poreux. Cependant, seulement l'effet de la combinaison de l'ajout du SPA avec $0.5 \%$ et des SCMs augmente la résistance chimique du composite $\mathrm{Pz}_{10} \mathrm{FS}_{5} \mathrm{Pl}_{0.5}$ vis-à-vis de cette attaque acide.

La Figure 3 montre l'effet de la combinaison de l'ajout du SPA et des SCMs sur les attaques acides des mortiers composites après 28 jours. A cette période d'immersion, on remarque pour l'ensemble des échantillons, que les pertes en masse dues à l'acide phosphorique sont généralement supérieures à celles dues à l'acide nitrique.

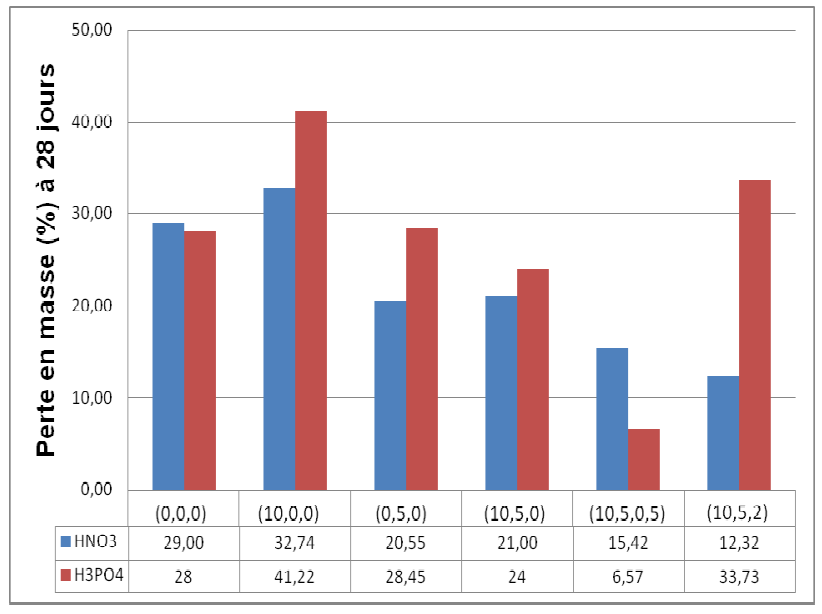

Fig. 3. Effet de la combinaison de l'ajout du SPA et des SCMs sur les attaques acides des mortiers composites après 28 jours.

Avec l'acide phosphorique, il $\mathrm{y}$ a formation $\mathrm{du}$ phosphate de calcium hydraté $\mathrm{Ca}_{3}\left(\mathrm{PO}_{4}\right)_{2} \cdot 6 \mathrm{H}_{2} \mathrm{O}, 0.002$ $\mathrm{g} / 100 \mathrm{ml}$ dans $\mathrm{H}_{2} \mathrm{O}$, qui est un sel peu soluble. Tandis qu'avec l'acide nitrique, la surface du mortier est recouverte du nitrate de calcium $\mathrm{Ca}\left(\mathrm{NO}_{3}\right)_{2} \cdot 2 \mathrm{H}_{2} \mathrm{O}$ (dihydraté) très soluble dans l'eau: $121.2 \mathrm{~g} / 100 \mathrm{ml}$ dans $\mathrm{H}_{2} \mathrm{O}$ et d'hydroxyde de fer qui est attribué au caractère oxydant de cet acide. Les réactions chimiques correspondantes sont indiquées dans les équations ( $1 \& 2$ ) ci-dessous. Ces résultats sont en accord avec ceux rapportés par Achoura et al. [16], Benosman et al. [17], Omrane et al. [18] et Belbachir et al. [19].

Les résistances mécaniques sont plus ou moins affectées par la concentration mais aussi et surtout par la nature des acides avec dans l'ordre le plus agressif d'après cette étude : l'acide $\mathrm{H}_{3} \mathrm{PO}_{4}$ puis l'acide nitrique $\mathrm{HNO}_{3}$ (Figure 3). On propose pour cela la séquence suivante: $\mathrm{H}_{3} \mathrm{PO}_{4}>\mathrm{HNO}_{3}$.

$2 \mathrm{HNO}_{3}+\mathrm{Ca}(\mathrm{OH})_{2} \rightarrow \mathrm{Ca}\left(\mathrm{NO}_{3}\right)_{2} \cdot 2 \mathrm{H}_{2} \mathrm{O}$

$2 \mathrm{H}_{3} \mathrm{PO}_{4}+3 \mathrm{Ca}(\mathrm{OH})_{2} \rightarrow \mathrm{Ca}_{3}\left(\mathrm{PO}_{4}\right)_{2} \cdot 6 \mathrm{H}_{2} \mathrm{O}$

Donc on peut conclure que les mortiers $\mathrm{FS}_{5}$ et $\mathrm{Pz}_{10} \mathrm{FS}_{5}$, les composites $\mathrm{Pz}_{10} \mathrm{FS}_{5} \mathrm{Pl}_{0.5}$ et $\mathrm{Pz}_{10} \mathrm{FS}_{5} \mathrm{Pl}_{2}$ ont bien résisté à l'attaque d'acide nitrique. Par contre, on a noté que le composite $\mathrm{Pz}_{10} \mathrm{FS}_{5} \mathrm{Pl}_{0.5}$ à mieux résister à l'attaque phosphorique.

L'influence positive du pourcentage des SCMs et du latex SPA sur la résistance chimique est traduite d'une part, par la réaction pouzzolanique qui fixe la portlandite libérée lors de l'hydratation du ciment pour former des gels de CSH supplémentaires de $2^{\text {ème }}$ génération et donc l'accroissement de la compacité des hydrates, engendrant une réduction de la porosité et d'autre part, par la formation du film du polymère SPA. Ce qui réduit la pénétration des agents agressifs. Ces résultats sont en accord avec ceux obtenus par Shaikh Ahmed [11], Belbachir et al. [19], Rossignolo et al. [20] et Monteny et al. [21].

\subsection{L'examen visuel}

L'inspection visuelle montre clairement l'état des différents spécimens avant et après attaques acides : 77 jours d'immersion dans $\mathrm{HNO}_{3}$ (Figure 4) et 49 jours dans $\mathrm{H}_{3} \mathrm{PO}_{4}$ à $8 \%$ (Figure 5).

Dans l'acide $\mathrm{HNO}_{3}$, (Figure 4): - les spécimens ne présentent pas une perte remarquable de leur forme, ils gardent toujours leur forme cubique. L'échantillon du témoin a un aspect plus dégradé (plus que la moitié) comparativement aux composites $\mathrm{Pz}_{10} \mathrm{FS}_{5} \mathrm{Pl}_{0.5}$ et $\mathrm{Pz}_{10} \mathrm{FS}_{5} \mathrm{Pl}_{2}$.

- La surface est recouverte d'une mince couche de rouille de couleur jaunâtre différente par rapport à la couleur initiale de la matrice cimentaire et qui semble due à la présence d'hydroxyde de fer $\mathrm{Fe}_{2} \mathrm{O}_{3}$ mal cristallisé [22].

- La surface des mortiers est très décapée avec un déchaussement important des grains de sable. La solubilité du sel formé, $\mathrm{Ca}\left(\mathrm{NO}_{3}\right)_{2} \cdot 2 \mathrm{H}_{2} \mathrm{O}$, après réaction de $\mathrm{HNO}_{3}$ avec la portlandite $\mathrm{Ca}(\mathrm{OH})_{2}$ libérée lors de l'hydratation du ciment et à sa grande lixiviation à l'extérieur avec décalcification progressive des C-S-H, engendrent ainsi des pertes de masse importantes.

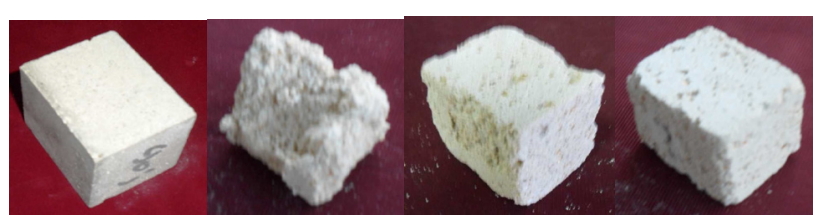

Fig. 4. Détérioration des spécimens après 77 jours d'immersion:

(1) témoin, (2) $(0 ; 0 ; 0) \mathrm{HNO}_{3}$, (3) $\left(\mathrm{Pz}_{10} \mathrm{FS}_{5} \mathrm{Pl}_{0.5}\right) \mathrm{HNO}_{3}$ et (4)

$\left(\mathrm{Pz}_{10} \mathrm{FS}_{5} \mathrm{Pl}_{2}\right) \mathrm{HNO}_{3}$, respectivement (du gauche à droite).

Dans l'acide $\mathrm{H}_{3} \mathrm{PO}_{4}$, (Figure 5): Réduction importante du volume des échantillons de mortier. En général, les surfaces du mortier sont plus détériorées dans $\mathrm{H}_{3} \mathrm{PO}_{4}$ que dans $\mathrm{HNO}_{3}$.

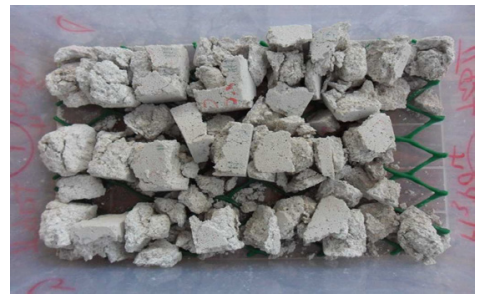

Fig. 5. Etat des spécimens après 49 jours d'immersion dans l'acide $\mathrm{H}_{3} \mathrm{PO}_{4}$.

Le taux d'agressivité de $\mathrm{H}_{3} \mathrm{PO}_{4}$ est supérieur à celui du $\mathrm{HNO}_{3}$. Ces constatations ont permis de confirmer les résultats obtenus par les essais des pertes de masse (Figures 1-3). 


\subsection{Caractérisation par Spectrométrie IRTF}

Le Tableau 4 et les Figures 6-8 présentent les positions et les intensités des bandes IRTF des spécimens \{mortier témoin, $\mathrm{Pz}_{10} \mathrm{FS}_{5} \mathrm{Pl}_{2}$ et $\mathrm{Pz}_{10} \mathrm{FS}_{5} \mathrm{Pl}_{0.5}$ \} exposés aux: milieu référence $(\mathrm{HR}=50 \pm 5 \%), \mathrm{HNO}_{3}$ et $\mathrm{H}_{3} \mathrm{PO}_{4}$. La superposition des différents spectres confirme:

- La formation de trace du nitrate de calcium et du phosphate de calcium hydraté après l'attaque par les acides $\mathrm{HNO}_{3}$ et $\mathrm{H}_{3} \mathrm{PO}_{4}$, respectivement, selon les réactions chimiques (Eq.1) \& (Eq.2);

- La décomposition totale de la portlandite par la solution agressive, d'où la disparition de sa bande majeure, selon les réactions chimiques (Eq.1) \& (Eq.2);

- La diminution de la quantité de la calcite $\mathrm{CaCO}_{3}$ par les différentes attaques acides.

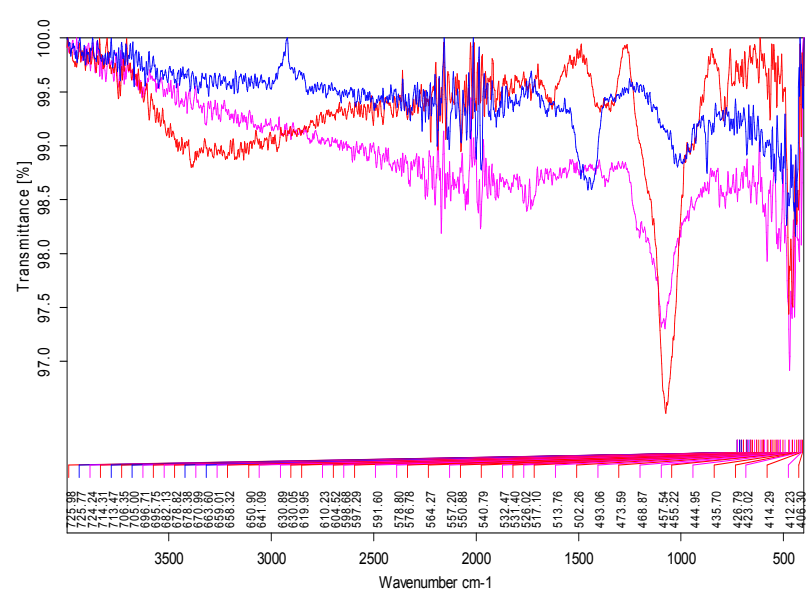

Fig. 6. Spectres IRTF du mortier $(0 ; 0 ; 0)$ dans les différents milieux: Bleu: Référence; Rouge: $\mathrm{HNO}_{3}$; Rose: $\mathrm{H}_{3} \mathrm{PO}_{4}$.

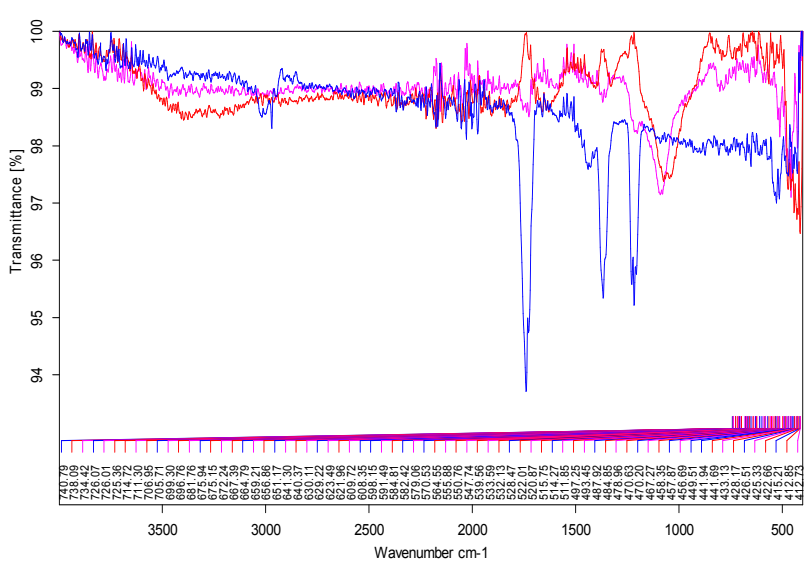

Fig. 7. Superposition des spectres IRTF du composite $\mathrm{Pz}_{10} \mathrm{FS}_{5} \mathrm{Pl}_{2}$ dans les différents milieux. Bleu: Référence sans attaque; Rouge: $\mathrm{HNO}_{3}$; Rose: $\mathrm{H}_{3} \mathrm{PO}_{4}$.

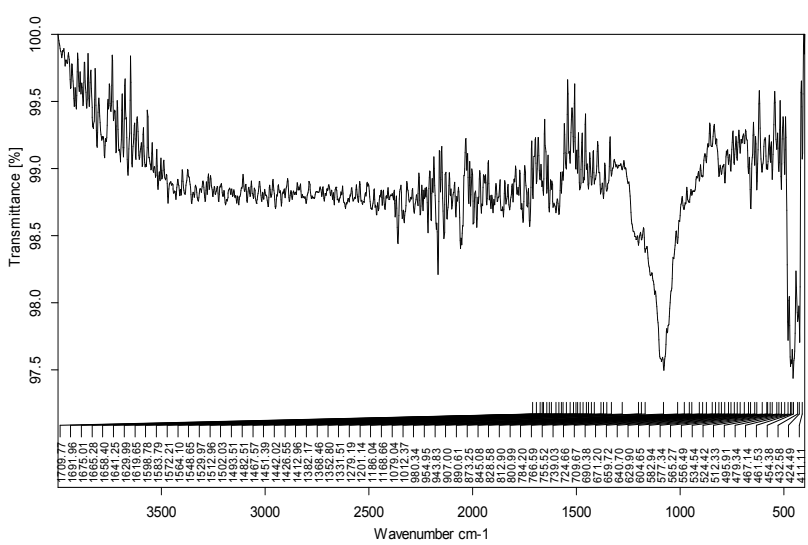

Fig. 8. Spectre IRTF du composite $\mathrm{Pz}_{10} \mathrm{FS}_{5} \mathrm{Pl}_{0.5}$ dans l'acide $\mathrm{H}_{3} \mathrm{PO}_{4}$.

Tableau 4. La Table infrarouge à transformée de Fourier des composites avant et après attaque par différents acides.

\begin{tabular}{|c|c|c|c|c|c|}
\hline \multicolumn{6}{|c|}{ Positions et Intensités des bandes d'absorption infrarouge (IRTF), $20^{\circ} \pm 1^{\circ} \mathrm{C}$} \\
\hline$\left(\mathrm{cm}^{-1}\right)$ & Groupes & Composés & Référence, $\mathrm{HR}=50 \pm 5 \%$ & $\mathrm{HNO}_{3}$ & $\mathrm{H}_{3} \mathrm{PO}_{4}$ \\
\hline$\sim 3000-3600$ & $\mathrm{H}_{2} \mathrm{O}, \mathrm{OH}$, liaison hydrogène & Gypse, CSH & +++ & + & + \\
\hline$\sim 3638$ (Fine) & $\mathrm{H}_{2} \mathrm{O}, \mathrm{OH}$, liaison hydrogène & Portlandite, $\mathrm{Ca}(\mathrm{OH})_{2}$ & +++ & 0 & 0 \\
\hline $3435-3452$ & $\mathrm{H}_{2} \mathrm{O}, \mathrm{OH}$ & AFm, Aft & ++ & + & + \\
\hline 1641-1654 & $\mathrm{H}_{2} \mathrm{O}, v \mathrm{O}-\mathrm{H}$ & $\mathrm{H}_{2} \mathrm{O}$ & + & + & + \\
\hline $1424-1431$ & $v \mathrm{C}-\mathrm{O}$ & $\mathrm{CaCO}_{3}$ (Calcite) & + & + & $+(\mathrm{F})$ \\
\hline $872-876$ & $v \mathrm{C}-\mathrm{O}$ & $\mathrm{CaCO}_{3}$ (Calcite) & + & $+(\mathrm{F})$ & $+(\mathrm{F})$ \\
\hline $710-714$ & $v \mathrm{C}-\mathrm{O}$ & $\mathrm{CaCO}_{3}$ (Calcite) & + & + & 1 \\
\hline$\sim 1010-1040$ & $v \mathrm{Si}-\mathrm{O}$ & Vibrations de CSH & $+(\mathrm{L})$ & $+(\mathrm{L})$ & $+(\mathrm{L})$ \\
\hline $1030-1080[23]$ & $v \mathrm{Ca}\left(\mathrm{NO}_{3}\right)_{2}$ & $\mathrm{Ca}\left(\mathrm{NO}_{3}\right)_{2} \cdot 2 \mathrm{H}_{2} \mathrm{O}$ & 0 & + & 0 \\
\hline $1350-1430$ [23] & $v \mathrm{Ca}_{3}\left(\mathrm{PO}_{4}\right)_{2}$ & $\mathrm{Ca}_{3}\left(\mathrm{PO}_{4}\right)_{2} \cdot 6 \mathrm{H}_{2} \mathrm{O}$ & 0 & 0 & ++ \\
\hline
\end{tabular}

F: Faible; L: Large; AFt: C(A,F). $3 \mathrm{CaSO}_{4} \cdot 32 \mathrm{H}_{2} \mathrm{O}$; AFm: $\mathrm{C}_{3} \mathrm{~A} \cdot 3 \mathrm{CaSO}_{4} \cdot 12 \mathrm{H}_{2} \mathrm{O}$.

\section{Conclusions}

Sur la base de l'étude de la durabilité des mortiers modifiés par l'effet de la combinaison du polymère SPA et des SCMs, on a pu tirer les conclusions suivantes :

○ La perte en masse des mortiers non modifiés immergés dans $\mathrm{HNO}_{3}$ ou $\mathrm{H}_{3} \mathrm{PO}_{4}$ est supérieure à celles des mortiers composites à base du SPA et des SCMs. La réduction des poids est due à la dissolution des hydrates formés qui mènent à des mortiers poreux.
○ On observe que les mortiers $\mathrm{FS}_{5}$ et $\mathrm{Pz}_{10} \mathrm{FS}_{5}$, les composites $\mathrm{Pz}_{10} \mathrm{FS}_{5} \mathrm{Pl}_{0.5}$ et $\mathrm{Pz}_{10} \mathrm{FS}_{5} \mathrm{Pl}_{2}$ ont bien résisté à l'attaque d'acide nitrique. Par contre, on a noté que le composite $\mathrm{Pz}_{10} \mathrm{FS}_{5} \mathrm{Pl}_{0.5}$ à mieux résister à l'attaque phosphorique.

- L'effet bénéfique de l'ajout du SPA comme addition au mortier modifié sur la durabilité des composites, à savoir $\mathrm{Pz}_{10} \mathrm{FS}_{5} \mathrm{Pl}_{0.5}$ et $\mathrm{Pz}_{10} \mathrm{FS}_{5} \mathrm{Pl}_{2}$. Ceci est dû à l'effet de la combinaison des additions minérales et de l'ajout du SPA. On a la formation du gel $\mathrm{CSH}$ de $2^{\text {ème }}$ génération par 
réaction pouzzolanique et la formation du film du Latex, respectivement.

- La résistance chimique des mortiers modifiés par l'effet de la combinaison de l'ajout du SPA et des SCMs à faible coût, et à faible impact environnemental, est améliorée vis-à-vis de l'attaque par les différents acides. Les résistances chimiques sont plus ou moins affectées par la concentration et par la nature des acides par ordre d'agressivité. On propose pour cela la séquence suivante : $\mathrm{H}_{3} \mathrm{PO}_{4}>\mathrm{HNO}_{3}$.

- L'identification des composés formés dans les mortiers composites avant et après attaque acide ont été suivies par spectrométrie IRTF.

Les meilleures propriétés de la durabilité de l'effet de la combinaison du polymère SPA et SCMs (PzN et FS), dans les mortiers modifiés observés dans cette étude, indiquent une durée de vie plus longue de la structure réparée en utilisant ce type de matériaux de réparation verts modifiés par un polymère.

Remerciements - Ce travail a été effectué avec l'apport financier du Ministère de l'Enseignement Supérieur et de la Recherche scientifique de l'Algérie, sous les subventions du projet CNEPRU B00L01UN310120130068. Nos plus vifs remerciements vont aussi à Mr M.T. GOUASMI et Mr O. HASNAOUI Directeur général du Groupe des Sociétés HASNAOUI, La TEKNACHEM Algérie.

\section{Références}

1. Y. Ohama, Hand book of polymer-modified concrete and mortars, properties and process technology. Noyes Publications, USA, (1995).

2. J. Baron, J.P. Olivier, Les Bétons Bases et Formulations. Association de l'industrie des liants hydrauliques, $2^{\text {ème }}$ Edition Eyrolles, (1997).

3. S. Chandra, Y. Ohama, Polymers in Concrete, CRC Press, Boca Raton, p 204, (1994).

4. E. Sakai, J. Sugita, Cem. Concr. Res. 25 [1] 127 (1995).

5. Y. Senhadji, G. Escadeillas, H. Khelafi, M. Mouli, A.S. Benosman, Eur. J. Environ. Civ. En. 16(1), 77 (2012).

6. J. Baron, J.P. Ollivier, La durabilité des Bétons. Presses de l'école nationale des ponts et chaussées, Paris, (1992).

7. Y. Senhadji, G. Escadeillas, M. Mouli, H. Khelafi, A.S. Benosman, Powder Technol. 254, 314 (2014).

8. M. Ghrici, S. Kenai, M. Said-Mansour, E-H. Kadri, J. Asian Archit. Build. Eng. 5(2), 349 (2006).

9. Y. Benali, F. Ghomari, J. Adhes. Sci. Technol. 31(3), 219 (2017).

10. B. Belbachir, A.S. Benosman, H. Taïbi, M. Mouli, Y. Senhadji, M. Belbachir. J. Mater. Environ. Sci. JMES, 7(2), 402 (2016).

11. Shaikh F.U. Ahmed, J. Mater. Civ. Eng. ASCE, 23(n9), 1311 (2011).

12. N. Latroch, A.S. Benosman, N. Bouhamou, B. Belbachir, Y. Senhadji, H. Taïbi, M. Mouli, Int. J. Eng. Res. in Africa JERA, 27, 27 (2016).
13. NF P 15-403. - Technique des essais. - Sable normal et mortier normal (homologuée le 31 juillet 1963) (1963).

14. EN 196-1, Methods of testing cement - Part 1: determination of strength. European Committee for Standardization, CEN, (2005).

15. ASTM C267-01, Standard Test Methods for chemical resistance of mortars, grouts, and monolithic surfacing and polymer concretes, Annual Book of ASTM Standards, (2012).

16. D. Achoura, Ch. Lanos, R. Jauberthie, B. Redjel, J. de Phys. IV France, EDP Sciences, 118, 159 (2004).

17. A.S. Benosman, M. Mouli, H. Taibi, M. Belbachir, Y. Senhadji, I. Bahlouli, D. Houivet, Environ. Eng. Manage. J., (EEMJ), 16(7), 1489 (2017).

18. M. Omrane, M. Mouli, A.S. Benosman, Y. Senhadji, Adv. Mater. Res. J., 1064, 3 (2015).

19. B. Belbachir, A.S. Benosman, H. Taïbi, Key Eng. Mater. KEM, 678, 123 (2016).

20. J.A. Rossignolo, M.V.C. Agnesini, Cem. Concr. Compos. 26, 375 (2004).

21. J. Monteny, N. De Belie, E. Vincke, W. Verstraete, L. Taerwe, Cem. Concr. Res. 31, 1359 (2001).

22. V. Pavlik, S. Uncik, Cem. Concr. Res. 28(6), 936, (1998).

23. Foil A. Miller, Charles H. Wilkins, Infrared Spectra and Characteristic Frequencies of Inorganic Ions; Their Use in Qualitative Analysis. Analytical Chemistry, 24, No. 8 (1952). 